\title{
ON GAUSSIAN SUMS
}

\author{
BY TAKASHI ONO
}

Communicated by G. D. Mostow, May 17, 1968

This note is an outline of some of the author's recent work on a generalization of Fourier transforms in adele spaces. Here we treat only the simplest case. The details and a generalization for an arbitrary ground $\boldsymbol{A}$-field and a system of polynomials will be given elsewhere. For the unexplained notions, see [1], [2] and [3].

Let $f(X)$ be an absolutely irreducible polynomial in $Q[X]$ $=Q\left[X_{1}, \cdots, X_{n}\right]$ such that the corresponding hypersurface $H$ $=\left\{x \in \Omega^{n} ; f(x)=0\right\}$ is nonsingular, where $\Omega$ denotes a universal domain containing $Q$. Let $V$ be the complement of $H$ in $\Omega^{n}$ viewed as an algebraic variety in $\Omega^{n+1}$ in an obvious way. Hence the $n$-form $\omega=f^{-1} d x, d x=d x_{1} \wedge \cdots \wedge d x_{n}$, is everywhere holomorphic and never zero on $V$. For each valuation $v$ of $\boldsymbol{Q}$, denote by $\boldsymbol{Q}_{v}$ the completion of $\boldsymbol{Q}$ at $v$. Denote by $A, A^{*}$ the adele ring and the idele group of $\boldsymbol{Q}$, respectively. For an idele $a \in A^{*},|a|_{A}$ will denote the module of $a$. The adelization $V_{A}$ of $V$ is then given by $V_{A}=\left\{x \in A^{n} ; f(x) \in A^{*}\right\}$. We denote by $\delta\left(Q_{n}^{n}\right), \delta\left(A^{n}\right)$ the space of Schwartz functions on $Q_{0}^{n}, A^{n}$, respectively. For each $v$, the $n$-form $\omega$ on $V$ induces a measure $\omega_{v}$ on $V_{Q_{0}}$ and we know that there is a well-defined measure $d V_{A}$ on $V_{A}$ of the form $\Pi_{0} \lambda_{0}^{-1} \omega_{v}$ with $\lambda_{\infty}=1$ and $\lambda_{p}=1-p^{-1}$. We know that the function

$$
Z(f, \phi, s)=\int_{V_{A}} \phi(x)|f(x)|_{A}^{*} d V_{A}, \quad \phi \in \delta\left(A^{n}\right),
$$

represents a meromorphic function for $\operatorname{Re} s>\frac{1}{2}$ having the single simple pole at $s=1$ with the residue $\int_{A^{n}} \phi(x) d A^{n}$, where $d A^{n}$ is the canonical measure on $A^{n}$ (cf. [4]).

Let $\chi$ be a basic character of $\boldsymbol{A}$ which identifies the additive group $A$ with its own dual and let $\chi_{0}$ be the similar character of the additive group $Q_{0}$ induced by $\chi$. For each $\xi \in A$ and $\phi \in \delta\left(A^{n}\right)$, the function $\phi_{\xi}(x)=\phi(x) \chi(f(x) \xi)$ is again in $\delta\left(A^{n}\right)$ and hence we have

$$
\operatorname{Res}_{s=1} Z\left(f, \phi_{\xi}, s\right)=\int_{A^{*}} \phi(x) \chi(f(x) \xi) d A^{n} \stackrel{\text { def. }}{=} \mathcal{G}_{f} \phi(\xi) .
$$

The transform $\phi \rightarrow \mathrm{g}_{f} \phi$ is a linear map of $S\left(A^{n}\right)$ into the space of con- 
tinuous functions on $\boldsymbol{A}$, which boils down to the Fourier transform $\phi \rightarrow \Im \phi$ when $n=1$ and $f(X)=X .^{1}$

Now, put $\eta=\operatorname{Res} \omega$, the residue form of $\omega$, this being an $(n-1)$-form on the hypersurface $H$ everywhere holomorphic and never zero. When the formal product $\Pi_{v} \eta_{v}$ (with no convergence factors) really defines a measure on $H_{A}$, we say that the canonical measure $d H_{A}=\Pi_{v} \eta_{v}$ exists on $H_{A}$. The classical theory of trigonometric sums suggests, at least formally, the equality

$$
\int_{\boldsymbol{A}} \mathcal{G}_{f} \phi d \boldsymbol{A}=\int_{H_{\boldsymbol{A}}} \phi d H_{A} \quad \text { for all } \phi \in \mathcal{S}\left(\boldsymbol{A}^{n}\right),
$$

where the right-hand side is essentially the singular series for $f(X)$ including the gamma factor. In view of (1), (2), one can interpret (3) as an equality connecting integrals on $V_{A}$ and $H_{A}$. More precisely, one can prove the following

THEOREM 1. If $f(X)$ satisfies the condition

$$
\mathcal{S}_{f} \phi \in L^{1}(\boldsymbol{A}) \text { for all } \phi \in S\left(\boldsymbol{A}^{n}\right),
$$

then the canonical measure $d H_{A}$ exists, $\phi \mid H_{A} \in L^{1}\left(H_{A}\right)$ for all $\phi \in \delta\left(A^{n}\right)$ and (3) holds.

Thus, the real problem is to find conditions on $f(X)$ so that (C) holds. For example, (C) is false for $f(X)=X_{1}^{2}+\cdots+X_{n}^{2}$ with $n \leqq 4$. Although we are still far from the complete solution of the problem, we can give the following sufficient conditions.

THEOREM 2. The condition (C) holds if $f(X)$ satisfies the following two conditions:

(I) $\operatorname{grad} f(x) \neq 0$ for all $x \in \Omega^{n}$,

(II) $\left|\sum_{x \in F_{p}^{n}} \zeta_{p^{f^{(p)}}(x)}\right| \leqq c p^{n-2-\epsilon}$ for almost all $p$, where $c, \epsilon$ are positive constants independent of $p, f^{(p)}(X)$ is the polynomial over the finite prime field $F_{p}$ obtained by reducing the coefficients of $f(X)$, for almost all $p$, and $\zeta_{p}$ is any one of the primitive pth roots of 1.

For example, $f(X)=X_{1}^{r_{1}} X_{2}^{r_{2}}+X_{1}+\sum_{i=3}^{n} X_{i}^{r_{i}}, r_{j} \geqq 2,1 \leqq j \leqq n$, satisfies (I), (II) whenever $n \geqq 7$.

REMARK 1. The condition (I) implies that $\operatorname{grad} f(x) \neq 0$ for all $x \in Q^{n}$, for all $v$. For this case one proves the stronger result:

1 Such a transform has been introduced by Weil in more general setting [3, Chapter I, No. 1]. For $p$-adic case, the evaluation of the transform is substantially the Gaussian sum for the polynomial $f(X)$. 


$$
\operatorname{Sos}_{f} \phi(\xi)=\int_{Q_{i}^{*}} \phi(x) \chi_{v}(f(x) \xi) d x_{v} \in S\left(Q_{v}\right) \text { for all } \phi \in S\left(Q_{v}^{n}\right) \text {. }
$$

This fact for $v=\infty$ has been suggested to us by Hörmander. We then found that the same is true for $v=p$.

REMARK 2. Unfortunately, the diagonal polynomial $f(X)$ $=\sum_{i=1}^{n} a_{i} X_{i}^{r_{i}}$ does not, in general, satisfy (I). A direct verification of the condition $(C)$ for such a polynomial seems to be not easy because arbitrary Schwartz functions are involved. However, (I) is intrinsic and this might be the case which must precede any attempt at a general theory.

\section{REFERENCES}

1. A. Weil, Basic number theory, Springer-Verlag, New York, 1967.

2. - Sur certains groupes d'opérateurs unitaires, Acta Math. 111 (1964), 143-211.

3. - Sur la formule de Siegel dans la thêorie des groupes classiques, Acta Math. 113 (1965), 1-87.

4. T. Ono, An integral attached to a hypersurface, Amer. J. Math. (to appear).

University of Pennsylvania, Philadelphia, Pennsylvania 19104 